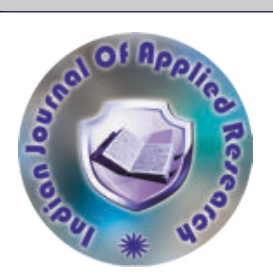

Radio Diagnosis

\title{
HEPATIC STEATOSIS IN LIVER DONORS: A COMPARATIVE STUDY BETWEEN COMPUTED TOMOGRAPHY AND MAGNETIC RESONANCE IMAGING - PROTON DENSITY FAT FRACTION BASED HEPATIC FAT GRADINGS.
}

Anandhu Krishnan
G*

Rajsekar C S

Srikanth Moorthy

\section{Rubalakshmi S}

Saitheja Paidipelly

\section{Sanju Sherji}

Fifiya.K.Yusuf
Department of Radiodiagnosis, Amrita Institute of Medical Sciences, Amrita School of Medicine, Amrita Vishwa Vidyapeetham, Cochin, Kerala, India. ${ }^{*}$ Corresponding Author Department of Radiodiagnosis, Amrita Institute of Medical Sciences, Amrita School of Medicine, Amrita Vishwa Vidyapeetham, Cochin, Kerala, India.

Department of Radiodiagnosis, Amrita Institute of Medical Sciences, Amrita School of Medicine, Amrita Vishwa Vidyapeetham, Cochin, Kerala, India.

Department of Radiodiagnosis, Amrita Institute of Medical Sciences, Amrita School of Medicine, Amrita Vishwa Vidyapeetham, Cochin, Kerala, India.

Department of Radiodiagnosis, Amrita Institute of Medical Sciences, Amrita School of Medicine, Amrita Vishwa Vidyapeetham, Cochin, Kerala, India.

Department of Radiodiagnosis, Amrita Institute of Medical Sciences, Amrita School of Medicine, Amrita Vishwa Vidyapeetham, Cochin, Kerala, India.

Department of Radiodiagnosis, Amrita Institute of Medical Sciences, Amrita School of Medicine, Amrita Vishwa Vidyapeetham, Cochin, Kerala, India.

Background: Living donor liver transplantation is being used as the main therapeutic option in the management of endstage liver disease patients especially since there is inadequate availability of deceased donors. Degree of hepatic steatosis in prospective liver donors can affect the tissue regeneration in both donors and recipients and as well as the success of the surgery. MRIPDFF is the best modality for the non-invasive assessment of hepatic steatosis in the literature, however CT is more commonly employed for the same. The qualitative data of hepatic fat using CT based methods are compared with MRI-PDFF based gradings.

Methods: The study included 35 prospective liver donors assessed between August 2018 to August 2020 . All patients were evaluated with MRIPDFF (IDEAL) sequence and fat fraction maps were obtained. The mean fat percentage was calculated and grading of hepatic steatosis was done. In plain MDCT $0.5 \mathrm{~mm}$ CT cuts, mean values of hepatic $\mathrm{CT}$ attenuation values corresponding MRI fat fraction maps were taken. Grading of the hepatic steatosis based on CT parameters like liver-spleen attenuation ratio (L/S) and liver-spleen attenuation difference(L-S) was done. Both CT and MRI based gradings were assessed for any statistical difference.

Results: The categorical data (grading) based on MR derived fat fraction and both CT based parameters mentioned above were evaluated with Chi Square test that showed no significant difference $(p=0.083)$.

Conclusion: Grading of hepatic steatosis using CT based parameters shows no statistically significant difference with MRI-PDFF based grading.

\section{KEYWORDS : MRI-PDFF (Magnetic Resonance Imaging - Proton Density Fat Fraction), CT (Computed Tomography).}

INTRODUCTION:

In the management of end-stage liver disease patients, liver transplantation is being used as a conclusive therapeutic option. Living donor liver transplantation (LDLT) is being progressively used as a safe, viable, and efficacious surgical procedure in the management of patients with liver failure (acute and chronic liver disease) and hepatocellular carcinoma. The role of imaging techniques in preoperative evaluation of a potential donor is vital as it aids not only in excluding focal or diffuse liver disease but also provides a thorough evaluation of the vascular as well as biliary anatomy along with estimation of liver volumes. This information is of prime importance before taking up such a key surgical task. This procedure involves the excision of the right hepatic lobe (segments V-VIII) from a healthy donor, without compromising the vascular supply or metabolic function of the residual left lobe (1). Liver procurement is possible because of its unique bi-lobar multi-segmental anatomy which aids surgeons to create grafts of varying size. Hepatic fat quantification is critical for donor selection in LDLT since graft steatosis may lead to increased risk of post-operative complications in both donor and recipient. In addition, it affects postoperative liver regeneration in the donor. Hepatic steatosis or excess fat accumulation within the intracellular vesicles, is the key histopathological feature of all cases of fatty liver, including non-alcoholic fatty liver disease (NAFLD) $(2,3)$. Plain CT imaging acts as a non-invasive modality in detecting hepatic steatosis by using the difference between hepatic and splenic attenuation values (L-S) or liver to spleen attenuation ratio (4), with radiation exposure being the main drawback (4). Proton density fat fraction (PDFF), quantified as the ratio of mobile protons (H1) belonging to triglyceride with respect to those to water, has been proposed as a standardized MR biomarker of steatosis (5). By design, MR-PDFF is an unconfounded and universal measure of triglyceride concentration, overcoming previously described flaws in conventional US, CT, and MRI (6). MRI-PDFF is the best modality for the noninvasive assessment of hepatic steatosis in the literature, however CT is the most commonly employed modality for donor assessment. MRIPDFF is already proven to be correlating with histopathology (7). Hence the statistical comparison between MRI-PDFF and CT gradings of hepatic steatosis is of high relevance.

\section{METHODS:}

Institutional review board approval was taken for this prospective study. Informed consent was taken from all patients before they underwent CT and MRI. The study period was from August 2018 to August 2020

\section{Study population:}

All prospective liver donors who underwent MDCT and MRI scan with PDFF sequence in the department of Radio-Diagnosis, Amrita Institute of Medical Sciences Research Centre, Kochi were assessed. MDCT was done followed by MRI with PDFF sequence. Interval between MDCT and MRI was less than a week. Patients with contraindications to MR or CT imaging, those who were not willing for CT or MRI-PDFF imaging, cirrhotic patients, those who were already diagnosed with hepatic space occupying lesions/ diffuse malignant infiltration/ storage disorders and those with history of multiple blood transfusions were excluded from the study.

Based on Pickhardt et al. (8) with 99.9\% confidence level and 90\% power, the minimum sample size came to 12 . In my study, a total of 35 cases where included.

Technical Information: 


\section{Techniques:}

3T Discovery MR750W MRI machine, GE Medical system, Milwaukee, WI and Philips Brilliance iCT 256 slice CT machine (Philips Health care, Cleveland, $\mathrm{OH}$ ) were used for the study. For 35 samples, MRI-PDFF imaging was performed on $3.0 \mathrm{~T}$ clinical MR systems (GE Healthcare) using a 12- or 32-channel (3.0 T) phased array torso coil. At $3.0 \mathrm{~T}$ acquisition parameters generally included $44 \times 44 \mathrm{~cm}$ field of view, $256 \times 160$ matrix, $8-\mathrm{mm}$ slice thickness, 32 slices, $3^{\circ}$ flip angle, $\pm 111 \mathrm{kHz}$ receiver bandwidth, $\mathrm{TR}=8.6 \mathrm{~ms}$, and 6 echoes (TEinit $=1.2 \mathrm{~ms}, \Delta \mathrm{TE}=1.0 \mathrm{~ms}$ ) acquired in two interleave echo trains of three echoes per repetition times (TR). Separated fat and water images were reconstructed using one of two region growing algorithms to avoid water-fat swapping, as well as perform spectral modelling of fat and T2* correction. Eddy current-related phase errors were addressed by using a hybrid magnitude/complex fitting technique. Auto-calibrated parallel imaging with a nominal 2D acceleration of 4 was used to reduce scan time to within a 20 second breath-hold. The MRI-PDFF images are calibrated to allow for direct region-of-interest (ROI) placement to derive an estimate of PDFF expressed as an absolute percentage $(0-100 \%)$. ROI $\left(100 \mathrm{~mm}^{2}\right)$ was placed in the desired seven segments of liver, avoiding large vessels and bile ducts, focal lesions and any obvious image artifacts. ROI placement was made side-by-side with CT to match ROI placement as closely as possible. All CT examinations were performed with multidetector CT (MDCT) device (256 iCT Philips). Filtered backprojection image reconstruction was typically with $5-\mathrm{mm}$ thickness at 3-mm intervals. As described above, a $100 \mathrm{~mm}^{2}$ ROI was placed in the desired hepatic segments and are co-registered manually with the ROI placed on the MRI-PDFF map, as closely as possible.

\section{Variables}

On unenhanced MDCT and MRI-PDFF, the following data were taken to consideration and analysis was done.

On MRI in IDEAL IQ fat only sequence, mean PDFF values are calculated from seven segments of liver with an ROI of $100 \mathrm{~mm}^{2}$.

(I) Mean attenuation values of liver attenuation values calculated from seven different segments with an ROI of $100 \mathrm{~mm}^{2}$.

(ii) Mean attenuation of seven splenic attenuation values was calculated from different sections with an ROI of $50 \mathrm{~mm}^{2}$. From this, Liver to Spleen attenuation difference values (L-S) and Liver to spleen attenuation ratio $(\mathrm{L} / \mathrm{S})$ were obtained.

\section{Following references of grading were adopted from Pickhardt et} al. (8):

Steatosis categories were defined as normal fat fraction less than 5\%, mild for $5 \%-14 \%$, moderate for $14 \%-28 \%$, and severe for greater than $28 \%$.

(ii) $\mathrm{L} / \mathrm{S}$ grading: Less than 0.8 - moderate to severe, 0.8 to 1.1 mild, greater than $1.1=$ normal

(iii) L-S grading: Greater than 5-Normal, 5 to -10 mild, less than -10moderate and severe. The grading of the hepatic fat was done based on liver-spleen CT attenuation ratio and liver-spleen CT attenuation values.

\section{STATISTICALANALYSIS:}

Categorical variables are presented using frequency and percentage. Continuous variables are presented using mean and standard deviation. Qualitative study was done by assessing the relationship between categorical data obtained from MR-PDFF and those obtained from CT through various parameters (Liver minus spleen attenuation and liver to spleen ratio). To measure the statistical significance of categorical data obtained from $\mathrm{CT}$ based fat fraction values and MRIPDFF values, Chi Square test was used. A p value of $<0.05$ was considered as statistically significant difference between the gradings.

\section{RESULTS:}

Total of 35 cases were taken for the study. The mean age of the patient involved in the study was $37.96 \pm 10.41$ years. There were 17 were males and 18 were females.

On grading the hepatic fat content on the samples using MRI-PDFF derived fat fraction values, $10(28.6 \%)$ were found to be having normal values, 18 (51.4\%) showed mild steatosis and $7(20 \%)$ showed moderate and above steatosis (Figure 1).

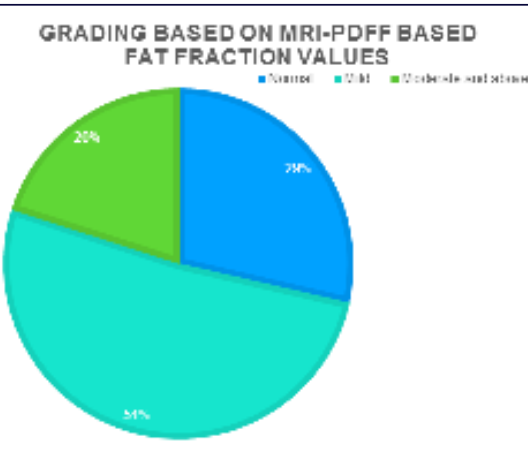

Figure 1 - Distribution of percentage of hepatic fat grading based on MRI-PDFF derived fat fraction values.

While assessing the grades of hepatic steatosis based on liver to spleen CT attenuation ratio (L/S), 13 (37.1\%) were categorized as normal values, $15(42.9 \%)$ showed mild steatosis and $7(20 \%)$ showed moderate and above steatosis (Figure - 1). The categorical data of grading obtained from MRI fat fraction and liver to spleen CT attenuation ratio (L/S) were evaluated with Chi-Square Test to look for any significant difference between the two gradings. The results showed that there was no difference between the categorical data with a p value of 0.083 (Table 1 ).

Table 1- Shows the statistical comparison between the qualitative data - Grading based on L/S and grading based on MRI-PDFF Fatfraction.

\begin{tabular}{|c|c|c|c|c|}
\hline $\begin{array}{c}\text { Grading based on CT } \\
\text { (L/S ratio) }\end{array}$ & \multicolumn{2}{|c|}{ MRI-PDFF Fat-fraction Grading } & P \\
\cline { 2 - 4 } & $\begin{array}{c}\text { Normal } \\
\mathrm{n}(\%)\end{array}$ & $\begin{array}{c}\text { Mild } \\
\mathrm{n}(\%)\end{array}$ & $\begin{array}{c}\text { Moderate and } \\
\text { above } \mathrm{n}(\%)\end{array}$ & \\
\hline Nolue & \\
\hline Mormal (13) & $10(76.9)$ & $3(23.1)$ & $0(0)$ & \multirow{2}{*}{0.083} \\
\hline Mild (15) & $0(0)$ & $15(100)$ & $0(0)$ & \\
\hline Moderate and above (7) & $0(0)$ & $0(0)$ & $7(100)$ & \\
\hline
\end{tabular}

Hepatic fat grading based on L/S ratio

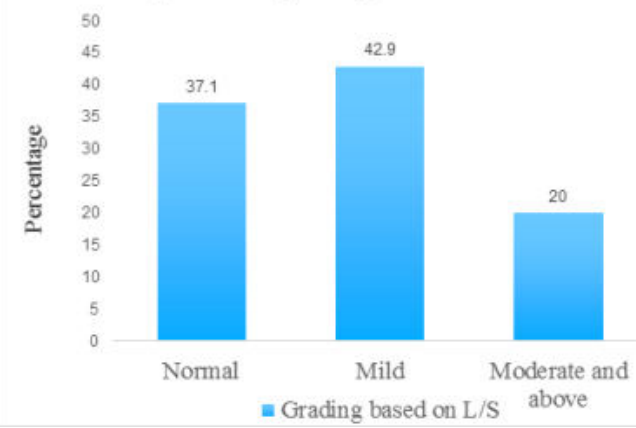

Figure 2 - Bar diagram showing categorical distribution of various grades of hepatic fat based on liver by spleen $(\mathrm{L} / \mathrm{S}) \mathrm{CT}$ attenuation values

While assessing the grades of hepatic steatosis based on liver to spleen CT attenuation difference (L-S), 13 (37.1\%) showed normal values, 15 $(42.9 \%)$ showed mild steatosis and $7(20 \%)$ showed moderate and above steatosis (Figure- 2). Both the categorical data of grading obtained from MRI derived fat fraction and liver to spleen CT attenuation difference (L-S) were evaluated with Chi-Square Test to look for any significant difference between the two gradings. The results showed that there was no significant difference between the two gradings with a p value of 0.083 (Table 2 )

Table 2 - Shows the statistical comparison and agreement between the qualitative data - Grading based on $L / S$ and grading based on MRI-PDFF Fat-fraction.

\begin{tabular}{|c|c|c|c|c|}
\hline \multirow{2}{*}{$\begin{array}{c}\text { Grading based on CT L-S } \\
\text { values }\end{array}$} & \multicolumn{3}{|c|}{ MRI-PDFF Fat-fraction Grading } & $\mathrm{P}$ \\
\hline & $\begin{array}{c}\text { Normal } \\
\mathrm{n}(\%)\end{array}$ & $\begin{array}{l}\text { Mild } \\
\mathrm{n}(\%)\end{array}$ & \begin{tabular}{|c|} 
Moderate and \\
above $\mathrm{n}(\%)$
\end{tabular} & Valu \\
\hline Normal (13) & $10(76.9)$ & $3(23.1)$ & $0(0)$ & 0.08 \\
\hline Mild (15) & $0(0)$ & $15(100)$ & $0(0)$ & \\
\hline Moderate and above (7) & $0(0)$ & $0(0)$ & $7(100)$ & \\
\hline
\end{tabular}



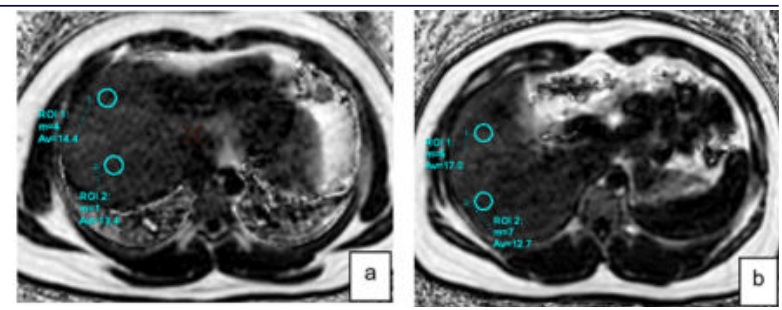

Figure 4: MRI PDFF Sequence (a) ROI of $100 \mathrm{~mm}^{2}$ is placed in segment VIII and VII of liver (b) ROI of $100 \mathrm{~mm}^{2}$ is placed in segment $V$ and VI of liver. Mean PDFF value - $>14 \%$ suggestive of moderate hepatic steatosis

Normal

Mild Moderate and above

Figure 3 - Shows categorical distribution of various hepatic fat grades using liver and spleen attenuation value difference $(L-S)$.

\section{DISCUSSION:}

Incidence of NAFLD is on the trend of rise both in the developed and in the developing countries due to the strong relationship between hepatic fat content with metabolic syndrome and cardiovascular disease. There can be subsequent progression of hepatic steatosis to non-alcoholic steato-hepatitis, chronic liver disease and HCC. Incidence of chronic liver disease and the need for liver transplant has been increasing in the Indian population predominantly due to its association with alcoholism, viral etiology and with an approximate number of around $800-1000$ LDLTs per year. Imaging modalities helps in assessing the vascular and biliary anatomy, fat quantification, donor liver volume assessment and to rule out any underlying lesions. The objective of the study is to assess whether there is any correlation between $C T$ and MRI-PDFF based qualitative hepatic fat quantification.

Grading of hepatic steatosis using a) MRI derived fat fraction and liver to spleen $\mathrm{CT}$ attenuation ratio (L/S) showed that there is no statistically significant difference between these gradings (categorical data) with a $\mathrm{p}$ value of 0.083 on Chi-Square test. Grading of hepatic steatosis using a) MRI derived fat fraction and liver to spleen CT attenuation difference (L-S) showed that there is no statistically significant difference between these gradings (categorical data) with a $p$ value of 0.083 on Chi-Square test. No other studies in the literature have simultaneously assessed both the various CT based gradings and MRIPDFF based gradings together for comparison.

Only correlational analysis has been done between the same in the limited available number of studies done on hepatic fat quantification. Zhe Guo et al., conducted a similar study showing significant negative correlation for L/S with MRI PDFF $\left(r=-0.837, r^{2}=0.70, p\right.$ value 0.002) (9) MRI derived fat fraction values and liver to spleen CT attenuation difference (L-S) values showed statistically significant negative correlation $\left(r=-0.828, r^{2}=0.68, p\right.$ value $\left.<0.001\right)$ Zhe Guo et al., conducted a similar study showing significant negative correlation for $\mathrm{L} / \mathrm{S}$ with MRI PDFF $\left(\mathrm{r}=0.854, \mathrm{r}^{2}=0.73\right.$, $\mathrm{p}$ value $\left.=0.03\right)(9)$.

Based on our study, hepatic steatosis, which is graded qualitatively based on various CT parameters described above and MRI-PDFF statistically show no significant difference. However, our study has the added benefit of having qualitative assessment with test of significance.
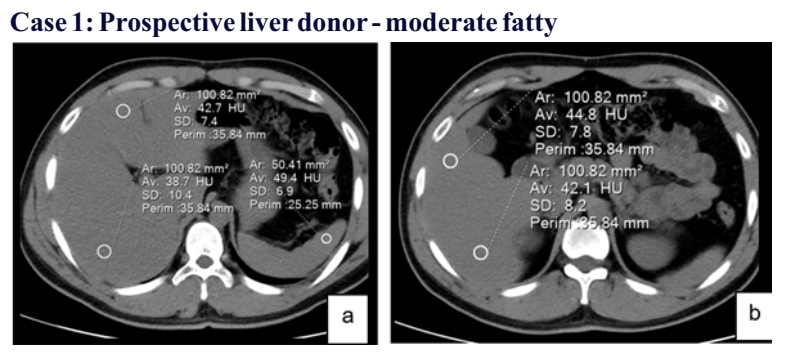

Figure 3: Unenhanced CT axial sections (a) ROI of $100 \mathrm{~mm} 2$ is placed in segment IVa and VII of liver and ROI of $50 \mathrm{~mm} 2$ in spleen (b) ROI of $100 \mathrm{~mm} 2$ is placed in segment $V$ and $V I$ of liver. Average $\mathrm{HU}$ less than $<42 \mathrm{HU}$, L-S: $<-10 \mathrm{HU}$ and $\mathrm{L} / \mathrm{S}:<0.8$ suggestive of moderate hepatic steatosis.

\section{Limitations:}

The minimum sample size calculated was 12. A sample size of 35 is almost three times the requirement. The studies defining frequency distribution in general population needs larger sample size. Samples of ROI taken from MRI Fat fraction map and corresponding CT images were from the same segments. No softwares were used to achieve synchronization. This would have increased the observer bias.

\section{CONCLUSION:}

Since there is no statistically significant difference between qualitative assessment of hepatic steatosis using both CT based methods and MRPDFF fat fraction, both non-invasive methods can be used for qualitative assessment of hepatic steatosis. As CT is better in assessing conveniently reliable method for assessing hepatic steatosis.

\section{REFERENCES}

1. Vohra, S., Goyal, N., \& Gupta, S. (2014). Preoperative CT evaluation of potential donors in living donor liver transplantation. The Indian journal of radiology \& imaging, 24(4), 350 . Burra, P. (2018). Nonalcoholic fatty liver disease and liver transplantation-Where do we stand?. World journal of gastroenterology, 24(14), 1491.

3. Zezos, P., \& Renner, E. L. (2014). Liver transplantation and non-alcoholic fatty liver disease. Worldjournal of gastroenterology: WJG, 20(42), 15532.

4. CL, C., CY, L., TY, C., TL, H., TY, L., CL, L., ... \& YH, W. (2001). Assessment of Donor Fatty Livers for Liver Transplantation. Transplantation 2001; 71: 1221. Transplantation, 71(9), 1206-1207.

5. Reeder, S. B., Hu, H. H., \& Sirlin, C. B. (2012). Proton density fat-fraction: a resonance imaging: JMRI, $36(5), 1011$

6. Serai, S. D., Dillman, J. R., \& Trout, A. T. (2017). Proton density fat fraction measurements at 1.5 -and 3-T hepatic MR imaging: same-day agreement among readers and across two imager manufacturers. Radiology, 284(1), 244-254.

7. Wang, X., Zhang, X., Ma, L., \& Li, S. (2017). Quantification of MRI-PDFF by complexbased MRI: phantom and rabbit study at 3.0 T. INTERNATIONAL JOURNAL OF based MRI: phantom and rabbit study at 3.0 T. INTERNATIONAL
CLINICALAND EXPERIMENTAL MEDICINE, 10(9), 13070-13080. Quantification of liver fat content with unenhanced MDCT: phantom and clinical correlation with MRI proton density fat fraction. American Journal of Roentgenology, 211(3), W151-W157.

9. Guo, Z., Blake, G. M., Li, K., Liang, W., Zhang, W., Zhang, Y., ... \& Pickhardt, P. J. Proton Density Fat Fraction: A Prospective Study of 400 Healthy Volunteers. Radiology, 294(1), 89-97. vascular anatomy and liver volume assessment, it can be used as a

2. Mikolasevic, I., Filipec-Kanizaj, T., Mijic, M., Jakopcic, I., Milic, S., Hrstic, I., ... \& standardized MR-based biomarker of tissue fat concentration. Journal of magnetic

8. Pickhardt, P. J., Graffy, P. M., Reeder, S. B., Hernando, D., \& Li, K. (2018). (2020). Liver Fat Content Measurement with Quantitative CT Validated against MRI 